\title{
Association Between Metabolic Syndrome and Peripheral Arterial Disease in Elderly Patients with Type 2 Diabetes
}

Lu Gao

Wei Zhao

Qi Liu

Mingzhao Qin

Department of Geriatrics, Beijing Tongren Hospital, Capital Medical University, Beijing, People's Republic of China
Correspondence: Lu Gao

Email audreygao@qq.com
Background: Peripheral artery disease (PAD) is a manifestation of atherosclerotic cardiovascular disease (ASCVD) and significantly increases the risk of myocardial infarction and stroke. As most patients with PAD are asymptomatic, this condition is largely neglected in clinical practice. Type 2 diabetes (T2DM) patients have higher prevalence of PAD. Therefore, early detection and intervention of diabetic PAD are very important. Metabolic syndrome (MetS) is a group of interrelated metabolic risk factors, a predictor of poor prognosis in elderly patients with ASCVD. Recently, many of the metabolic risk factors as well as the overall concept of MetS itself have sparked a great deal of debate regarding their precise roles in PAD.

Objective: To evaluate the relationship between metabolic syndrome (MetS) and peripheral arterial disease (PAD) in elderly patients with type 2 diabetes (T2DM).

Methods: Two hundred and eighty-one elderly T2DM patients admitted to Beijing Tongren Hospital from October 2016 to December 2017 were divided into PAD group $(n=136)$ and non-PAD (NPAD) group $(\mathrm{n}=145)$. Their medical records, physical examination parameters and laboratory testing parameters were later recorded and analyzed by multivariate logistic regression analysis.

Results: No significant difference was detected in general clinical data and laboratory testing parameters between the two groups $(\mathrm{P}>0.05)$. The incidence of MetS was significantly higher in PAD group than in NPAD group $(88.2 \%$ vs $70.3 \%, \mathrm{P}=0.001)$. Multivariate logistic regression analysis showed that the risk of PAD was 1.762-fold higher in MetS patients after adjustment for age, sex, history of smoking and alcohol consumption, WC, SBP, serum TC and HbAlc level, eGFR and duration of DM (95\% CI: 1.205-6.330, $\mathrm{P}=0.016)$.

Conclusion: MetS closely correlates with PAD in elderly T2DM patients, though no significant difference has been detected in their metabolic indicators.

Keywords: elderly, type 2 diabetes, metabolic syndrome, peripheral arterial disease

\section{Introduction}

Peripheral artery disease (PAD) is one of the most common types of atherosclerotic cardiovascular disease (ASCVD). Although most patients with PAD are asymptomatic, their risk of getting myocardial infarction and stroke rises dramatically. Worse still, their risk of death within 10 years is more than six times as high as that of non-PAD patients. ${ }^{1}$ Patients with type 2 diabetes (T2DM) have higher prevalence of PAD, coupled with earlier occurrence and more rapid progress of cardiovascular and cerebrovascular diseases. Their amputation rate, perioperative mortality and total mortality are also increased. ${ }^{2}$ Therefore, early detection and 
intervention of diabetic PAD have important clinical significance in improving the quality of life in patients and reducing their incidence of serious complications.

Metabolic syndrome (MetS), a group of interrelated metabolic risk factors including impaired glucose regulation, hypertension, central obesity and dyslipidemia, is known to be associated with pathological changes in blood vessels. In China, individuals with MetS are three to ten times more likely to develop ASCVD. ${ }^{3}$ MetS is a predictor of poor prognosis in elderly patients with cardiovascular and cerebrovascular diseases.

In recent years, many of the metabolic risk factors, and even the overall concept of MetS itself, have sparked numerous debates regarding their precise roles in PAD. ${ }^{4,5}$ The purpose of this study is to discuss whether the presence of MetS is associated with PAD and to evaluate the independent effect of MetS on PAD in elderly patients with T2DM.

\section{Materials and Methods Study Population}

Participants of this study were T2DM patients aged 60 or above, selected from geriatric wards in Beijing Tongren Hospital between October 2016 and December 2017. Exclusion criteria for the participants were as follows: patients with acute infection, autoimmune diseases, endstage nephropathy, severe cardiac dysfunction [NYHA grade III-IV], malignant tumor, undergone arterial vascular reconstruction of lower extremity, or with the ankle-brachial index (ABI) more than 1.4 (at least one limb). Finally, 281 participants were available for the follow-up test that divided them into two groups, 136 with PAD and 145 without.

\section{Data Collection}

All participants received a standardized examination. Data regarding age, sex, smoking, alcohol consumption and history of diabetes and hypertension were obtained with detailed medical records. Body Mass Index (BMI) was calculated as weight $(\mathrm{kg})$ divided by height squared $\left(\mathrm{m}^{2}\right)$. Waist circumference (WC) was measured at the level of the umbilicus in $\mathrm{cm}$. Blood pressure (BP) was measured three times when participants were seated, and the average of the last two measurements was adopted. Blood samples were collected after an overnight fast for the determination of plasma glucose, HbAlc, total cholesterol (TC), triglycerides (TG), high-density lipoprotein cholesterol (HDL-C), low-density lipoprotein cholesterol (LDL-C) concentrations and serum creatinine (Scr). GFR was estimated by using the modified MDRD formula for Chinese patients: ${ }^{6}$ eGFR $\left(\mathrm{mL} / \mathrm{min} / 1.73 \mathrm{~m}^{2}\right)=$ $175 \times \mathrm{Scr}^{-1.234} \times \mathrm{age}^{-0.179} \times 0.79$ (if female) .

\section{Definition of Clinical and Biochemical Variables}

According to the criteria recommended by 2011 guidelines of American Heart Association Foundation/American Heart Association, PAD was diagnosed with ankle brachial index $\leq 0.90$ in either leg. ${ }^{7}$ Diagnosis of hypertension was based on meeting any of three criteria: systolic blood pressure (SBP) of $\geq 140 \mathrm{mmHg}$, diastolic blood pressure (DBP) of $\geq 90$ mmHg or current use of antihypertensive drugs. ${ }^{8}$ Central obesity is based on WC cutoff $\geq 85 \mathrm{~cm}$ in women and $\geq 90 \mathrm{~cm}$ in men according to the guideline for the prevention and treatment of type 2 diabetes mellitus in China (2020 edition). ${ }^{9}$ Overweight and obesity are respectively identified as BMI $24-28 \mathrm{~kg} / \mathrm{m}^{2}$ and BMI equal to or over $28 \mathrm{~kg} / \mathrm{m}^{2}$ according to the Working Group on Obesity in China (WGOC). ${ }^{2002,10}$ Smokers were defined as those who had smoked $\geq 1$ cigarette/day for at least 1 year. Drinkers were defined as those who had consumed $\geq 30 \mathrm{~g}$ of alcohol/week on average for at least 1 year.

\section{Assessment of $A B I$}

ABI was measured and calculated by a VaSera VS-1000 (Fukuda Denshi Co., Tokyo, Japan) vascular screening system. SBP measurements in the bilateral brachial, dorsalis pedis, and posterior tibial arteries were obtained in the supine position using a hand-held Doppler instrument with a $5-\mathrm{mHz}$ probe (CBM304) after a five-minute rest. The sphygmomanometer cuff airbag (manufactured by Sanofi company in France) was $10 \mathrm{~cm}$ wide and $40 \mathrm{~cm}$ long. Brachial artery pressures were averaged to obtain the ABI denominator. To avoid potential bias from subclavian stenosis, when the two brachial artery pressures differed by $10 \mathrm{mmHg}$ or more, the highest brachial artery pressure was used as the denominator. For each lower extremity, the ABI numerator was the highest pressure (dorsalis pedis or posterior tibial) from that leg. ${ }^{11}$

\section{Definition of MetS}

According to the definition of the guideline for the prevention and treatment of type 2 diabetes mellitus in China (2020 edition), ${ }^{9}$ MetS is diagnosed when three or more of the following risk factors are present: a. abdominal obesity 
Table I Clinical Characteristics of Studied Subjects with and without PAD

\begin{tabular}{|c|c|c|c|c|}
\hline & NPAD & PAD & $\mathbf{t} / \mathbf{z} / \chi^{2}$ & P-value \\
\hline $\mathbf{n}$ & 145 & 136 & & \\
\hline Age (year) & $67.77 \pm 7.02$ & $70.94 \pm 7.43$ & $-4.56 \mid$ & 0.522 \\
\hline Female $[n(\%)]$ & $77(53.1)$ & $72(52.9)$ & 0.000 & 0.986 \\
\hline Smoking [n(\%)] & $43(29.7)$ & $48(35.3)$ & 0.387 & 0.534 \\
\hline Alcohol [n(\%)] & $20(13.8)$ & $20(14.7)$ & 0.011 & 0.918 \\
\hline FPG (mmol/l) & $6.73(5.52,8.75)$ & $7.11(6.37,9.76)$ & -1.332 & 0.186 \\
\hline HbAlc (\%) & $8.38 \pm 1.74$ & $8.87 \pm 2.00$ & -1.427 & 0.501 \\
\hline Duration of diabetes (year) & $|0.38 \pm 6.6|$ & $10.87 \pm 6.74$ & 0.991 & 0.322 \\
\hline SBP (mmHg) & $|33.9| \pm \mid 7.45$ & $138.06 \pm 20.84$ & -1.199 & 0.181 \\
\hline DBP (mmHg) & $78.22 \pm 8.19$ & $76.79 \pm 8.68$ & 0.903 & 0.600 \\
\hline Hypertension [n(\%)] & $93(66.9)$ & $108(81.8)$ & 2.811 & 0.094 \\
\hline TC (mmol/l) & $4.62 \pm 0.99$ & $4.48 \pm 0.75$ & 0.778 & 0.055 \\
\hline TG (mmol/l) & $1.46(1.11,2.10)$ & $1.60(1.03,2.35)$ & -0.758 & 0.449 \\
\hline HDL-C (mmol/l) & $1.16 \pm 0.31$ & $1.10 \pm 0.35$ & 0.922 & 0.485 \\
\hline LDL-C (mmol/l) & $2.93 \pm 0.83$ & $2.77 \pm 0.72$ & 1.005 & 0.333 \\
\hline Hyperlipidemia [n(\%)] & $93(64.1)$ & $88(64.7)$ & 0.004 & 0.950 \\
\hline WC $(\mathrm{cm})$ & $89.69 \pm 9.51$ & $92.58 \pm 8.23$ & -1.636 & 0.210 \\
\hline Central obesity [n(\%)] & 91 (62.8) & $100(73.5)$ & $\mathrm{I} .40 \mathrm{I}$ & 0.237 \\
\hline BMI $\left(\mathbf{k g} / \mathbf{m}^{2}\right)$ & $24.8 I \pm 3.23$ & $25.37 \pm 3.69$ & -0.878 & 0.857 \\
\hline Generalized obesity [n(\%)] & $25(17.2)$ & $24(17.6)$ & 0.003 & 0.955 \\
\hline eGFR $\left[\mathrm{mL} /\left(\min \cdot 1.73 \mathrm{~m}^{2}\right)\right]$ & $105.19 \pm 66.14$ & $86.92 \pm 26.53$ & 1.578 & $0.54 I$ \\
\hline
\end{tabular}

Notes: Data are means \pm SE, median (P25-P75) or raw numbers (\%). Continuous data were used for univariate general linear models and categorical data were analyzed by $\chi^{2}$ tests.

Abbreviations: PAD, peripheral arterial disease; NPAD, non-peripheral arterial disease; FPG, fasting plasma glucose; HbAIc, glycated haemoglobin; SBP, systolic blood pressure; DBP, diastolic blood pressure; TC, total cholesterol; TG, triglycerides; HDL-C, high-density lipoprotein; LDL-C, low-density lipoprotein cholesterol; WC, waist circumference; BMI, body mass index; eGFR, estimated glomerular filtration rate.

(WC $\geq 85 \mathrm{~cm}$ in women and $\geq 90 \mathrm{~cm}$ in men); b. level of fasting $\mathrm{TG} \geq 1.70 \mathrm{mmol} / \mathrm{l}$; c. level of fasting HDL-C: $<1.04$ $\mathrm{mmol} / \mathrm{l}$; d. SBP $\geq 130 \mathrm{mmHg}$ or $\mathrm{DBP} \geq 85 \mathrm{mmHg}$ on examination and/or when the participant had a prior diagnosis of hypertension and was receiving antihypertensive medications; and e. $\mathrm{FPG} \geq 6.1 \mathrm{mmol} / \mathrm{l}$ or $2 \mathrm{~h} \mathrm{PG} \geq 7.8 \mathrm{mmHg}$ after oral glucose tolerance test and/or when the participant had a prior diagnosis of diabetes mellitus and was receiving antidiabetic medications.

\section{Statistical Analysis}

All statistical analyses were conducted with the software package SPSS version 22.0 for Windows. For the continuous variables with a normal distribution, mean $\pm \mathrm{SD}$ was reported and the independent $t$-test was used to compare subjects of the two groups. For the discrete variables or the continuous variables without a normal distribution, the median (P25-P75) was reported, and a Mann-Whitney rank test was used to examine the differences between the groups. In the meantime, distribution of discrete/qualitative variables was compared by Pearson chi-square test. Multivariable logistic regression analysis was used to estimate crude and adjusted odds ratios (ORs) (95\% CIs) to allow for differences between groups with respect to demographic and risk factors and control for potentially confounding variables. A p-value of less than 0.05 was considered statistically significant.

\section{Results}

A total of 281 T2DM patients with $(n=136)$ or without $(\mathrm{n}=145)$ PAD comprised the study groups, with 132 men and 149 women aged between 60 and 85 (68.92 \pm 7.47$)$.

The demographic and biochemical parameters of the two groups are shown in Table 1. There were no statistical differences between PAD group and NPAD group in clinical characteristics (age, sex, duration of diabetes, levels of FPG, HbAlc, SBP, DBP, TC, TG, HDL-C, LDL-C, WC, BMI and eGFR and the proportion of smoking, alcohol, central obesity, hypertension and dyslipidemia).

When patients were grouped according to the number of detected components of the MetS; however, the percentage of patients with PAD increased linearly with the increasing number of components in their respective groups $(0 \%, 32.0 \%, 40.7 \%, 54.1 \%, 69.2 \%$ respectively), 
A

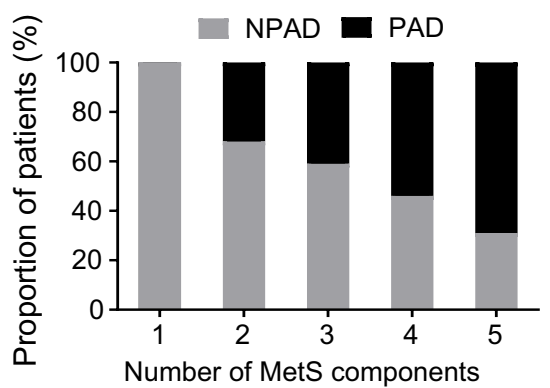

B

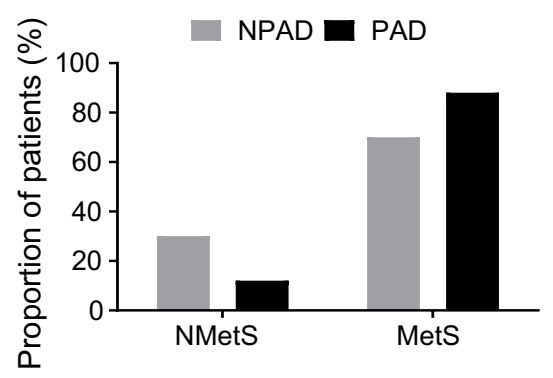

Figure I The association of PAD with MetS.

Notes: (A) Prevalence (\%) of patients with PAD in relation to number of MetS components (I to 5). I represents elevated glucose, while 2 to 5 represent elevated glucose combined with one to four other components of MetS, respectively. (B) Comparison of prevalence of PAD between NMetS and MetS groups.

and the percentage of patients without PAD decreased with the increasing number of components $(100.0 \%, 68.0 \%$, $59.3 \%$, 45.9\%, 30.8\% respectively) (Pearson $\chi^{2}=25.691$, $\mathrm{P}=0.001$ ), as is illustrated in Figure 1A. Of the 59 subjects without MetS, 16 had PAD (27.1\%). However, in MetS group involving 222 subjects, the number was 120 (54.1\%). The inclination to PAD in MetS group was thus significantly higher than in NMetS group $\left(\chi^{2}=13.541\right.$, $\mathrm{P}=0.001$ ), as is illustrated in Figure 1B.

Next, we performed logistic regression analysis to identify the risk of PAD with the number of components of MetS. The risk for PAD increased considerably with the increment of MetS components. The aggregation of elevated glucose combining three or four other metabolic factors in the MetS increased 2.162 times [odds ratio (95\% CI): 3.162 (1.594-6.270), $\mathrm{P}=0.001]$ or 5.047 times [odds ratio (95\% CI): $6.047(2.657-13.761), \mathrm{P}=0.001$ ] than those with elevated glucose only (Figure 2A). To determine if there is an independent association between PAD and MetS, we performed multivariable logistic regression analysis and found that MetS [OR (95\% CI): 3.162 (1.681-5.947), $\mathrm{P}=0.001$ ] was closely associated with PAD. With an adjustment for age, sex and the proportion of smoking and alcohol, MetS was significantly associated with and an independent risk factor for PAD [odds ratio (95\% CI): 2.875 (1.494-5.531), $\mathrm{P}=0.002$ ]. Moreover, such association was not affected by an additional adjustment for WC, SBP, TC, HbA1c, eGFR and duration of diabetes [odds ratio (95\% CI): 2.762 (1.205-6.330), $\mathrm{P}=0.016$ ] (Figure 2B).

\section{Discussion}

PAD is a manifestation of atherosclerotic disease, with $80 \%$ of its patients being asymptomatic. Regardless of the presence of symptoms, the morbidity and mortality of cardiovascular and cerebrovascular diseases were
A

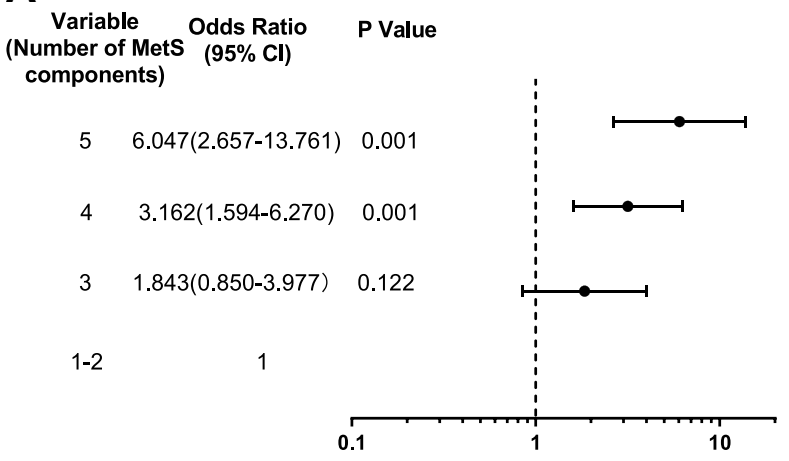

B

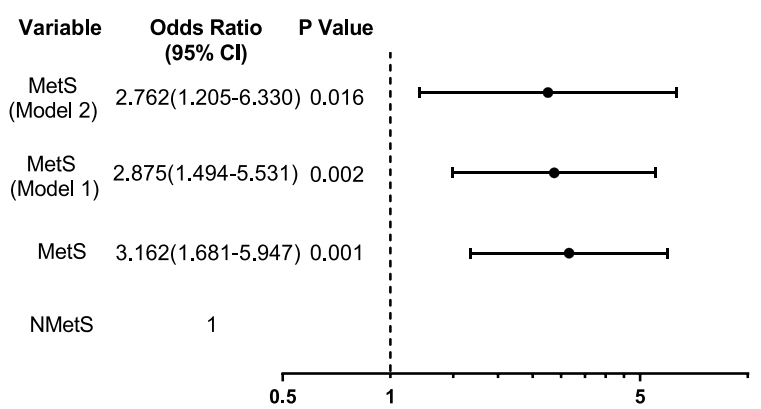

Figure 2 Contribution of cumulative metabolic components in PAD and Odds ratios for PAD with/without MetS.

Notes: Binary logistic regression was conducted to assess the association of PAD with the number of components of MetS (A) and MetS (B) using the Entry method; adjusted odds ratios (ORs) and the $95 \%$ confidence intervals ( $\mathrm{Cls}$ ) given. I-5 were identified as the number of components of MetS in (A), with I being elevated glucose as a referent and 2-5 elevated glucose combining one to four other factors of the MetS. The group without MetS was used as a referent in (B). Adjustment variables included the basic confounders (age, sex, smoking and alcohol) in Model I. In Model 2, WC, SBP, TC, HbAIc, eGFR and duration of diabetes were also considered as other adjustment variables and were thus added to Model I. 
significantly higher in them. ${ }^{12-16}$ Clinical trials have confirmed that $\mathrm{ABI}$ measurement is a simple, non-invasive and effective method for the diagnosis of PAD, whose prevalence varies from $3.4 \%$ to $42 \%$ depending on age, sex, ethnicity and other conditions. ${ }^{17-19}$ In China, the first large-scale multicenter survey about PAD involving more than 10,000 patients with diabetes reported that the prevalence of diabetic PAD was $21.2 \% .{ }^{17}$ In our study, however, the figure for elderly T2DM patients was $48.4 \%$, which should arouse enough attention from clinicians. It is therefore of great clinical significance to understand the incidence of senile diabetic PAD and analyze the related risk factors for its prevention and treatment.

In our study, T2DM patients with eGFR $<60 \mathrm{~mL} / \mathrm{min} /$ $1.73 \mathrm{~m}^{2}$ and severe cardiac dysfunction [NYHA grade IIIIV] were excluded specifically to avoid the confounding factors of renal and cardiac insufficiency, which were associated with increased risks of PAD. We subsequently found that most metabolic indices in PAD group were slightly higher than those in NPAD group, but there were no significant differences in a number of biological or laboratory tests between the two groups. However, after defining each component of the MetS according to the criteria, we found that the proportion of MetS in PAD group was remarkably higher than that in NPAD group. Furthermore, the presence of MetS was significantly associated with and an independent risk factor for PAD.

MetS is a series of pathophysiological changes, including glycolipid metabolism disorder, insulin resistance, vascular endothelial dysfunction, inflammation and increased sympathetic nerve activity. ${ }^{20-24}$ It is closely related to the incidence of atherosclerotic diseases which directly lead to the increase in the morbidity and mortality of ASCVD. ${ }^{25}$ Moreover, each MetS component can directly promote vascular injury and atherosclerosis formulation; meanwhile, there is also a synergistic effect among them, as they can interact and influence each other. For this reason, the interplay between two or more MetS components can aggravate vascular lesions. In this study, with the increase in the number of MetS components, the risk of PAD went up. When there were four or more MetS components, the risk of PAD was considerably higher than that of patients with only one or two metabolic abnormalities. When MetS was taken as a whole index, the proportion of MetS in PAD group was also significantly higher than that in NPAD group. It is therefore suggested that in addition to metabolic abnormalities, the combination of metabolic factors can also influence the occurrence and development of PAD.

Intensive, multifactorial interventions can effectively reduce the incidence of macrovascular and microvascular complications in T2DM patients. ${ }^{26}$ Our research also showed that it is insufficient to control one single risk factor of blood glucose for diabetic PAD. Instead, we should pay attention to other potential metabolic risk factors at the same time before carrying out targeted, intensive and multifactorial intervention for some modifiable risk factors.

Our study has several limitations that must be taken into account. Firstly, this study is based on hospitalized patients, which may have unpredictable selection bias. Secondly, due to vascular calcifications, some patients may have a falsely normal ABI that covers their true PAD identity, but they are missed using the test. Thirdly, this study has yet to consider the use of drugs of hypoglycemic, antihypertensive and statins and levels of inflammatory biomarkers, which will have a certain impact on the occurrence and development of PAD. Fourthly, retrospective case-control studies cannot evaluate the causal relationship directly; therefore, our findings may suggest that MetS is an indicator, but not predictor of PAD. Finally, participants are small in number for a crosssectional study. The adverse effects of MetS on diabetic PAD should be further confirmed in a larger cohort study based on different ethnic populations using different MetS definitions. However, we have no reasons to believe these would substantially bias the associations reported herein.

In conclusion, with the increase in the number of metabolic risk factors, the risk of PAD increases accordingly. Patients with multiple metabolic risk factors are more likely to suffer from PAD. Even though there is no significant difference in the level of metabolic indicators in elderly T2DM patients, MetS, as a metabolic disorder syndrome, is closely associated with and a strong, independent indicator of PAD. Timely diagnosis of MetS can provide valuable information for the prevention and control of PAD.

\section{Data Sharing Statement}

The data are available on reasonable request from the corresponding author.

\section{Ethics Statement}

The study was conducted with the approval from the Ethics Committee of Beijing Tongren Hospital, Capital Medical University, and adhere to the tenets of the 
Declaration of Helsinki. Written informed consent was obtained from each participant.

\section{Acknowledgments}

The authors would like to thank all the health-care workers and patients participated in the study. The authors thank Di Zeng from the University of Beijing Language and Culture for language editing.

\section{Funding}

There is no funding for this study.

\section{Disclosure}

The authors report no conflicts of interest in this work.

\section{References}

1. Aboyans V, Criqui MH, Abraham P, et al. Measurement and interpretation of the ankle-brachial index: a scientific statement from the American Heart Association. Circulation. 2012;126(24):2890-2909. doi:10.1161/CIR.0b013e318276fbcb

2. Brownrigg JR, Schaper NC, Hinchliffe RJ. Diagnosis and assessment of peripheral arterial disease in the diabetic foot. Diabet Med. 2015;32(6):738-747. doi:10.1111/dme.12749

3. Nestel P, Lyu R, Low LP, et al. Metabolic syndrome: recent prevalence in East and Southeast Asian populations. Asia Pac J Clin Nutr. 2007;16(2):362-367.

4. Brevetti G, Schiano V, Sirico G, Giugliano G, Laurenzano E, Chiariello M. Metabolic syndrome in peripheral arterial disease: relationship with severity of peripheral circulatory insufficiency, inflammatory status, and cardiovascular comorbidity. J Vasc Surg. 2006;44(1):101-7;discussion 7. doi:10.1016/j.jvs.2006.02.048

5. Sumner AD, Khalil YK, Reed JF 3rd. The relationship of peripheral arterial disease and metabolic syndrome prevalence in asymptomatic US adults 40 years and older: results from the national health and nutrition examination survey (1999-2004). J Clin Hypertens. 2012;14(3):144-148. doi:10.1111/j.1751-7176.2011.00580.x

6. Ma YC, Zuo L, Chen JH, et al. Modified glomerular filtration rate estimating equation for Chinese patients with chronic kidney disease. J Am Soc Nephrol. 2006;17(10):2937-2944. doi:10.1681/asn.2006 040368

7. Rooke TW, Hirsch AT, Misra S, et al. 2011 ACCF/AHA focused update of the guideline for the management of patients with peripheral artery disease (updating the 2005 guideline): a report of the American College of Cardiology Foundation/American Heart Association Task Force on Practice Guidelines. $J$ Am Coll Cardiol. 2011;58(19):2020-2045. doi:10.1016/j.jacc.2011.08.023

8. James PA, Oparil S, Carter BL, et al. 2014 evidence-based guideline for the management of high blood pressure in adults: report from the panel members appointed to the Eighth Joint National Committee (JNC 8). JAMA. 2014;311(5):507-520. doi:10.1001/jama.201 3.284427

9. Society CD. Guideline for the prevention and treatment of type 2 diabetes mellitus in China (2020 edition). J Clin Endocrinol Metab. 2021;37(4):311-398. doi:10.3760/cma.j.cn311282-20210304-00142.

10. Zhou BF. Predictive values of body mass index and waist circumference for risk factors of certain related diseases in Chinese adultsstudy on optimal cut-off points of body mass index and waist circumference in Chinese adults. Biomed Environ Sci. 2002;15 (1):83-96.
11. Vidula H, Liu K, Criqui MH, et al. Metabolic syndrome and incident peripheral artery disease - the multi-ethnic study of atherosclerosis. Atherosclerosis. 2015;243(1):198-203. doi:10.1016/j. atherosclerosis.2015.08.044

12. Ford ES. Risks for all-cause mortality, cardiovascular disease, and diabetes associated with the metabolic syndrome: a summary of the evidence. Diabetes Care. 2005;28(7):1769-1778. doi:10.2337/ diacare.28.7.1769

13. Eberhardt RT, Coffman JD. Cardiovascular morbidity and mortality in peripheral arterial disease. Curr Drug Targets Cardiovasc Haematol Disord. 2004;4(3):209-217. doi:10.2174/1568006043336230

14. McDermott MM, Guralnik JM, Tian L, et al. Baseline functional performance predicts the rate of mobility loss in persons with peripheral arterial disease. J Am Coll Cardiol. 2007;50(10):974-982. doi:10.1016/j.jacc.2007.05.030

15. Fowkes FG, Murray GD, Butcher I, et al. Ankle brachial index combined with Framingham risk score to predict cardiovascular events and mortality: a meta-analysis. JAMA. 2008;300(2):197-208. doi:10.1001/jama.300.2.197

16. McDermott MM, Guralnik JM, Tian L, et al. Associations of borderline and low normal ankle-brachial index values with functional decline at 5-year follow-up: the WALCS (Walking and Leg Circulation Study). J Am Coll Cardiol. 2009;53(12):1056-1062. doi:10.1016/j.jacc.2008.09.063

17. Zhang X, Ran X, Xu Z, et al. Epidemiological characteristics of lower extremity arterial disease in Chinese diabetes patients at high risk: a prospective, multicenter, cross-sectional study. $J$ Diabetes Complications. 2018;32(2):150-156. doi:10.1016/j.jdiacomp.2017.1 0.003

18. Wen J, Yang J, Shi Y, et al. Comparisons of different metabolic syndrome definitions and associations with coronary heart disease, stroke, and peripheral arterial disease in a rural Chinese population. PLoS One. 2015;10(5):e0126832. doi:10.1371/journal.pone.0126832

19. Aronow WS. Management of peripheral arterial disease of the lower extremities in elderly patients. J Gerontol a Biol Sci Med Sci. 2004;59(2):172-177. doi:10.1093/gerona/59.2.m172

20. Alberti KG, Eckel RH, Grundy SM, et al. Harmonizing the metabolic syndrome: a joint interim statement of the international diabetes federation task force on epidemiology and prevention; National Heart, Lung, and Blood Institute; American Heart Association; World Heart Federation; International Atherosclerosis Society; and International Association for the Study of Obesity. Circulation. 2009;120(16):1640-1645. doi:10.1161/circulationaha.109.192644

21. Garg PK, Biggs ML, Carnethon M, et al. Metabolic syndrome and risk of incident peripheral artery disease: the cardiovascular health study. Hypertension. 2014;63(2):413-419. doi:10.1161/hypertensio naha.113.01925

22. Biscetti F, Porreca CF, Bertucci F, et al. TNFRSF11B gene polymorphisms increased risk of peripheral arterial occlusive disease and critical limb ischemia in patients with type 2 diabetes. Acta Diabetol. 2014;51(6):1025-1032. doi:10.1007/s00592-014-0664-1

23. Biscetti F, Pitocco D, Straface G, et al. Glycaemic variability affects ischaemia-induced angiogenesis in diabetic mice. Clin Sci. 2011;121 (12):555-564. doi:10.1042/cs20110043

24. Flex A, Biscetti F, Iachininoto MG, et al. Human cord blood endothelial progenitors promote post-ischemic angiogenesis in immunocompetent mouse model. Thromb Res. 2016;141:106-111. doi:10.1016/j. thromres.2016.03.012

25. Piazzolla G, Candigliota M, Fanelli M, et al. Hyperhomocysteinemia is an independent risk factor of atherosclerosis in patients with metabolic syndrome. Diabetol Metab Syndr. 2019;11(1):87. doi:10.1186/s13098-019-0484-0

26. van Leiden HA, Dekker JM, Moll AC, et al. Risk factors for incident retinopathy in a diabetic and nondiabetic population: the Hoorn study. Arch Ophthalmol. 2003;121(2):245-251. doi:10.1001/archopht.12 1.2.245 


\section{Publish your work in this journal}

Diabetes, Metabolic Syndrome and Obesity: Targets and Therapy is an international, peer-reviewed open-access journal committed to the rapid publication of the latest laboratory and clinical findings in the fields of diabetes, metabolic syndrome and obesity research. Original research, review, case reports, hypothesis formation, expert opinion and commentaries are all considered for publication. The manuscript management system is completely online and includes a very quick and fair peer-review system, which is all easy to use. Visit

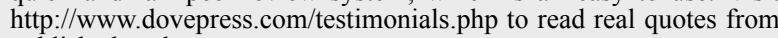
published authors. 